University of Nebraska - Lincoln

DigitalCommons@University of Nebraska - Lincoln

PreColumbian Textile Conference VIII /

Jornadas de Textiles PreColombinos VIII (2019)

Centre for Textile Research

$6-2020$

\title{
La cestería de los cazadores-recolectores, procedente de la cueva de la Candelaria, Torreón, Coahuila, México
}

Gloria Martha Sánchez Valenzuela

Alejandra Quintanar Isaías

Ana Jaramillo Pérez

Follow this and additional works at: https://digitalcommons.unl.edu/pctviii

Part of the Art and Materials Conservation Commons, Fiber, Textile, and Weaving Arts Commons, Indigenous Studies Commons, Latin American Languages and Societies Commons, Museum Studies Commons, and the Other History of Art, Architecture, and Archaeology Commons

This Article is brought to you for free and open access by the Centre for Textile Research at DigitalCommons@University of Nebraska - Lincoln. It has been accepted for inclusion in PreColumbian Textile Conference VIII / Jornadas de Textiles PreColombinos VIII (2019) by an authorized administrator of DigitalCommons@University of Nebraska - Lincoln. 


\title{
La cestería de los cazadores-recolectores, procedente de la cueva de la Candelaria, Torreón, Coahuila, México
}

\author{
Dra. Gloria Martha Sánchez Valenzuela ${ }^{1}$ \\ Dra. Alejandra Quintanar Isaías ${ }^{2}$ \\ Mtra. Ana Jaramillo Pérez ${ }^{3}$
}

1. Laboratorio de Conservación de Material Arqueológico de Origen Orgánico - Coordinación Nacional de Conservación Del Patrimonio Cultural del Instituto Nacional de Antropología e Historia. gloriamsv.cncpc@inah.gob.mx

2. Laboratorio de Anatomía Funcional y Biomecánica de Plantas Vasculares. Departamento de Biología -Universidad Autónoma Metropolitana Iztapalapa. aqi@xanum.uam.mx

3. Laboratorio de Anatomía Funcional y Biomecánica de Plantas Vasculares. Departamento de Biología -Universidad Autónoma Metropolitana Iztapalapa. jaramillo@xanum.uam.mx

\section{Resumen}

En 1953 se realiza la primera exploración arqueológica al Norte de la República Mexicana por parte del Instituto Nacional de Antropología e Historia (INAH), que conformó un grupo interdisciplinario para explorar la Cueva de la Candelaria, ubicada en el Valle de las Delicias en el estado de Coahuila. Esta cueva es una de las más importantes de México, dónde se logró recuperar -gracias a su característica de cueva seca- un vasto patrimonio arqueológico de origen orgánico que nos permite conocer mejor a los grupos de cazadores-recolectores que habitaron la región.

Dentro de la gran diversidad de objetos se encontraron un número considerable de cestas o canastas rígidas elaboradas con la técnica de enrollado en espiral (Sánchez Valenzuela, Gloria M; 2017:119), de diversos tamaños de forma cóncava y cónica. El estudio e identificación de los materiales permitieron ratificar la maestría y conocimiento que tenían los grupos de cazadores recolectores para aprovechar los materiales que les proporcionaba su entorno elaborando artefactos que utilizaban cotidianamente.

A través del estudio anatómico microscópico se identificaron las fibras empleadas en la manufactura de las cestas, provenientes del xilema secundario de plantas del género Jatropha sp. perteneciente a la familia Euphorbiaceae, y abundantes en la región de la Laguna.

La reconstrucción de estas técnicas puede tener impacto en la visión que se tiene de estos grupos nómadas: su tecnología, el conocimiento y uso de los recursos naturales de esta región.

Palabras Clave: Cestería, Cazadores-recolectores, fibras, Cueva de la Candelaria

\begin{abstract}
In 1953, the National Institute of Anthropology and History (INAH) carried out the first archaeological exploration to the North of the Mexican Republic, which formed an interdisciplinary group to explore the Cueva de la Candelaria, located in the Valle de las Delicias in the state of Coahuila. This cave is one of the most important in Mexico, where it was able to recover a vast archaeological heritage of organic origin that allows us to know better the groups of hunter-gatherers who inhabited the region.
\end{abstract}

DOI: $10.32873 /$ unl.dc.zea.1212

Published in PreColumbian Textile Conference VIII / Jornadas de Textiles PreColombinos VIII, ed. Lena Bjerregaard and Ann Peters

(Lincoln, NE: Zea Books, 2020). https://digitalcommons.unl.edu/zeabook/ 
Among the great diversity of objects found there are a considerable number of rigid baskets elaborated with the technique of spiral winding (Sánchez Valenzuela, Gloria M; 2017:119), of various sizes, both concave and conical. The study and identification of the materials allowed us to ratify the mastery and knowledge that groups of hunter-gatherers employed to take advantage of the materials provided by their environment by making artifacts that they used on a daily basis.

Through the microscopic anatomical analyses, it was possible to identify the plant species of the fibers with which the baskets were manufactured. These are secondary xylem sheafs of the genus Jatropha dioica belonging to the Euphorbiaceae family; species abundant in the Laguna region.

The reconstruction of these techniques can enrich the current vision of these nomadic groups: their technology, knowledge and use of the natural resources of this region.

Keywords: Basketry, Hunter-gatherers, fibers, Candelaria Cave.

\section{Résumé}

En 1953 est réalisée la première exploration archéologique du Nord de la République Mexicaine par l'Institut National d'Anthropologie et d'Histoire (INAH), qui a réuni un groupe interdisciplinaire pour explorer la Cueva de la Candelaria, localisée dans la Vallée de las Delicias dans l'Etat de Coahuila. Cette grotte est l'une des plus importantes du Mexique, où l'on a pu récupérer - grâce à sa caractéristique de grotte sèche - un vaste patrimoine archéologique d'origine organique qui nous permet de mieux connaître les groupes de chasseurs-cueilleurs qui ont habité la région.

Dans la grande diversité d'objets, ont été découvert un nombre considérable de paniers, ou corbeilles rigides, élaborées selon la technique de l'enroulé en spirale (Sánchez Valenzuela 2017 : 119), de diverses tailles et de forme concave ou conique. L'étude et l'identification des matériaux ont permis de ratifier la maîtrise et la connaissance que les groupes de chasseurscueilleurs avaient pour tirer profit des matières premières que fournissait leur environnement en produisant des artefacts qu'ils utilisaient quotidiennement.

Grâce à l'étude anatomique, il a été possible d'identifier les fibres utilisées dans la vannerie. Ces dernières, provenant du xylème secondaire des plantes du genre Jatropha sp., et appartenant à la famille des Euphoribiaceae ; sont abondantes dans la région de la Laguna.

La reconstruction de ces techniques peut enrichir la vision actuelle que nous avons des groupes nomades : leur technologie, leur connaissance et l'utilisation des ressources naturelles de cette région.

Mots-clés: Vannerie, chasseurs-cueilleurs, fibres, Cueva de la Candelaria.

\section{Introducción}

La cueva de la Candelaria está situada en la Comarca Lagunera, al pie de la sierra de la Candelaria, en el valle de las Delicias al suroeste del estado de Coahuila, México.

La cueva se abre a unos 3om sobre el nivel del bolsón y el orificio para ingresar es tipo chimenea; la cámara principal mide aproximadamente un metro de diámetro, el tiro se ensancha de forma irregular tanto transversal como verticalmente y se aproxima a la forma rómbica. "La cueva de la Candelaria nunca fue un sitio de habitación con acumulación estratigráfica de depósitos culturales, sino una simple grieta de grandes dimensiones, utilizada por los antiguos laguneros como un enorme depósito mortuorio" (González Arratia, Arqueología Mexicana Vol. 30, 1998:63)

La Cueva de la Candelaria fue descubierta por casualidad en 1953 por personas oriundas de la región quienes dieron aviso al Instituto Nacional de Antropología e Historia (INAH), que decide realizar allí el primer proyecto formal de investigación en el norte de México.
El proyecto implicó varias temporadas de campo con un equipo interdisciplinario conformado por: Manuel Maldonado Koerdell (geólogo y paleontólogo), Arturo Romano (antropólogo físico), Francisco González Rul (arqueólogo) y Pablo Martínez del Río (antropólogo).

Cabe resaltar que en 1953 la región norte de México era una zona poco estudiada; se sabía que estuvo habitada por grupos de cazadores recolectores y que existían numerosas cuevas que fueron utilizadas como casa habitación o bien como sitios de enterramiento. La escasa información existente, para el conocimiento de dichos grupos, era a través de los informes que anualmente rendían los padres misioneros a sus superiores Jesuitas (Aveleyra, Martínez del Río, Pablo; 1956:25-31).

El desarrollo del proyecto permitió conocer mejor a las culturas del norte: sus sistemas de enterramiento, alimentación, materiales empleados, flora, fauna; así como ciertas enfermedades que se observaron en algunos de los cráneos dónde son evidentes las lesiones sifilíticas (Pijoan Aguadé, 2000), así como el extraordinario trabajo sobre tecnología 
textil de la Dra. Johnson (1977). Los objetos descubiertos permanecieron, una parte en un pequeño museo de la Escuela Preparatoria Venustiano Carranza (EPVC) y otra en el antiguo Museo Nacional de Antropología. En 1976, algunos de los objetos del antiguo Museo se trasladaron a las instalaciones del nuevo Museo Regional de la Laguna (MUREL) y hasta el 2013 se incorporan los que estaban albergados en la Escuela Preparatoria. Por lo anterior muchos materiales quedaron pendientes de estudio y análisis como es el caso de la cestería.

Actualmente la colección de la Cueva de la Candelaria se encuentra resguardada, principalmente en dos depósitos: en el Museo Regional de la Laguna, (Torreón, Coahuila) y en el Museo Nacional de Antropología en la Ciudad de México, lugares en los que se ha podido tener acceso e iniciar estudios formales sobre los diversos objetos rescatados.

Los objetos de cestería encontrados son representaciones fundamentales de la vida cotidiana de los pueblos nómadas del norte. Se trata de cuencos u ollas manufacturados con materia prima poco convencional; excepcionales en el tejido y en el posible uso, dado que se trata de objetos que podrían haber sido empleados como contenedores de líquidos o para procesar alimentos. Sus formas sugieren una tecnología emblemática para la época dado que estos grupos nómadas tendrían necesidades de almacenamiento y transporte posiblemente de granos $\mathrm{u}$ otras materias primas en contenedores que al ser ligeros facilitarían su movilidad.

El estudio de la materia prima vegetal y la tecnología con la que se manufacturaron estos objetos puede dar luz sobre el gran conocimiento del uso de los recursos vegetales que tenían esos grupos humanos para resolver y procurar su subsistencia, por lo que el presente trabajo se ha enfocado a la descripción y estudio de la manufactura de estos objetos y enfatizando el tipo de material con el que fueron elaboradas.

\section{Antecedentes}

\section{Características del emplazamiento}

La cueva de la Candelaria se sitúa en la región cultural denominada de los Irritila o laguneros, dónde habitaron grupos nómadas dedicados a la pesca, recolección y caza. Vivían cerca de lugares con aprovisionamiento de agua y manufacturaban sus artefactos con los materiales que les proporcionaba su entorno, ambiente desértico caracterizado por bruscas variaciones de temperatura que han moldeado el tipo de vegetación y fauna de esa región.

La característica del contexto de la cueva estable y seco, permitió que en el interior se conservaran bultos mortuorios y materiales asociados a ellos; de acuerdo con Romano
(1953:4-12), los cadáveres de adultos e infantes fueron colocados en posición fetal dentro de grandes mantos donde eran envueltos y atados con cuerdas para asegurarlos.

El paquete funerario estaba formado por los restos humanos, acompañados de objetos que les habrían pertenecido como son utensilios, herramientas y vestimenta: navajas, raspadores, punzones, collares, pendientes. Así como tejidos diversos: redes, petates, enredos de cabeza, yahuales, sandalias, bolsas de pescador, bandas, lienzos y una gran variedad de cestos o canastas de diversos tamaños.

Según Luis Aveleyra los objetos denotan una larga ocupación de la zona y por consiguiente implican cierto sedentarismo, es muy posible que la cultura candelariense abarque un lapso del 1000 al 1600 d.C. La cronología se obtuvo por los materiales arqueológicos asociados y por dos fechas de radiocarbono 14 (Aveleyra,1964), una de un fragmento de textil que tiene una edad que fluctúa entre los años 1095 a 1315 d.C. y la otra de un hueso humano que dio una fecha entre 1100 y 1300 d.C. (Pijoan, Mansilla, 2000:213).

\section{Materiales y métodos en el estudio de las cestas}

\section{Descripción general de los objetos.}

Las cestas estudiadas proceden de la colección de la Cueva de la Candelaria bajo resguardo del Museo Regional de la Laguna, Torreón, Coahuila. Se trata de objetos tejidos con un material vegetal muy resistente, dada la época en la que pudieron construirse. Presentan un sistema horizontal de varillas que se amarran con un sistema vertical, usándose para ello fibras del mismo material vegetal. Estos objetos pueden presentar o no un recubrimiento negro en su interior y exterior. La presencia del recubrimiento sugiere una práctica para sellar la porosidad del tejido y servir como contenedor o recipiente de líquidos. Aquellas que no lo presentan cuentan con una decoración sencilla pintada de color rojo, sugiriendo otro tipo de función.

El estado de conservación de las cestas proporcionadas para este estudio es regular ya que presentan fibras quebradizas, con deformaciones, pérdida de tejido y de capa de recubrimiento; así como grietas, fisuras, manchas, polvo y tierra acumulada.

Es relevante señalar que entre los objetos estudiados por un lado se encuentran cestas que han perdido el fondo, y por el otro fragmentos de fondos sueltos que sugiere podrían ser correspondientes entre ambos.

También se observa que existen varias cestas con remiendos originales sobre todo en el área de la base o fondo, lo que sugiere una práctica de reparación y recuperación de este tipo de utensilios dada la complejidad en la manufactura, el procesamiento de la materia prima y posiblemente de la disponibilidad de la materia prima. 


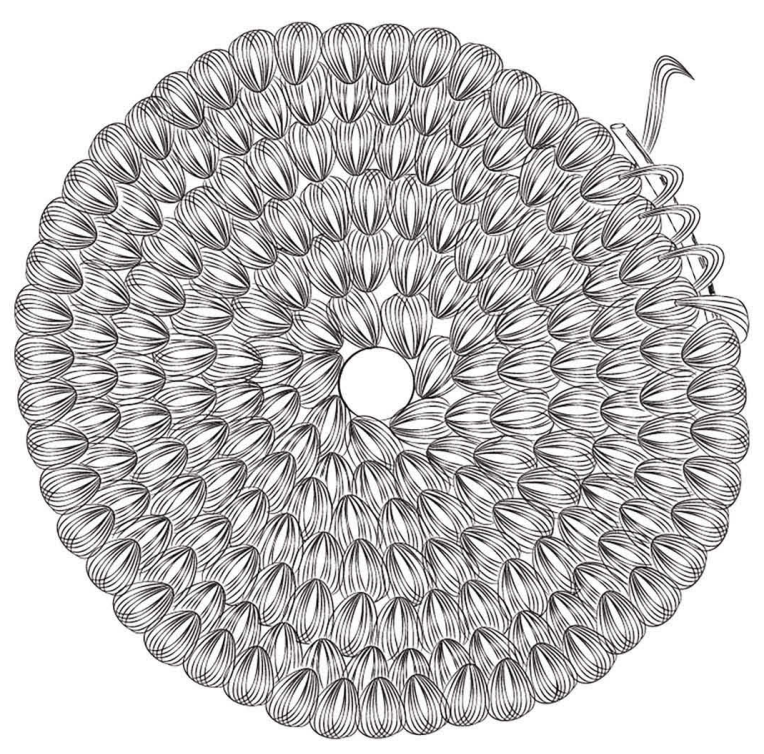

Figura 1a. Esquema de la técnica de enrollado en espiral.

\section{Descripción del tejido.}

Dentro de los bienes reguardados en el MUREL se localizan 15 cestas completas (algunas sin fondo), 11 fondos y varios fragmentos de cestería que tienen como común denominador estar elaborados con fibras rígidas o semiduras con la técnica de enrollado en espiral (coiled basketry).

De acuerdo con Adovasio (1977), las cestas están constituidas por dos elementos equivalentes a la trama y urdimbre de los textiles; denominando base o fundación a los elementos o fibras que se enrollan horizontalmente conformando una estructura en espiral, y puntos de sutura o sujeción a los elementos o fibras que se desarrollan verticalmente para unir los elementos horizontales a través de puntadas como se observa en las figuras $\mathbf{1 a} \mathbf{y} \mathbf{1 b}$.

Una vista general del trabajo de manufactura (figura 2a) permite observar cómo se unen la fundación con los puntos de sujeción, resultando un tejido que mantiene una estructura firme y cerrada. Los dos elementos unidos conforman el tejido dando estructura a las cestas (figura $\mathbf{2 b}$ ), lo que es relevante pues dado el origen del material, las fibras verticales no rajan el haz de fibras horizontales, por el contrario, garantizan una mejor sujeción. En la figura $2 c$ se muestra un corte transversal del tejido destacando una médula o región central conformada por un haz de fibras leñosas inicial sin huecos colocadas horizontalmente, sujetas de forma vertical, con el mismo material, dando soporte y resistencia al tejido.

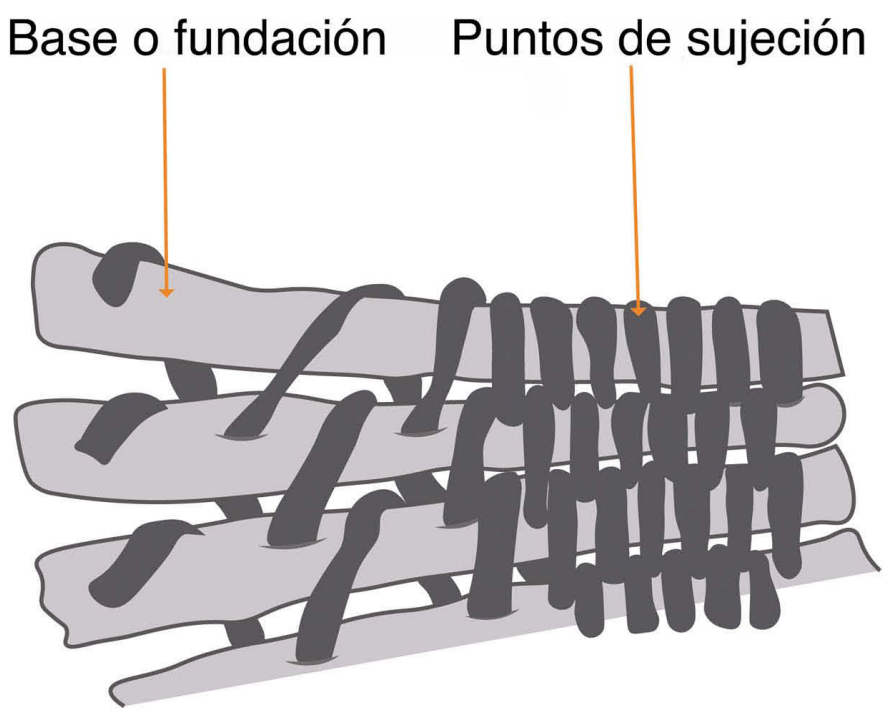

Figura 1b. Descripción de elementos de la técnica del tejido enrollado en espiral. Dibujos: Castro Rodríguez, Miriam; CNCPC-INAH, 2019.

3. Descripción del tejido empleando fibras xilemáticas.

La fundación está conformada por haces o paquetes de fibras xilemáticas con un diámetro de $5 \mathrm{~mm}$ de espesor aproximadamente, que conforman la estructura de la cesta y funcionan como las paredes que le dan resistencia. Aunque las fibras son semirrígidas al provenir del tallo de la planta, son notablemente flexibles, lo que permite dar la curvatura al enrollado en espiral.

Los puntos de sujeción están conformados por haces largos de fibras xilemáticas de aspecto lustroso, de un espesor de entre 1-2 mm, con los que se logra ejecutar muchas inserciones para sujetar, tensar y tejer amplias secciones a lo largo de la fundación. Los puntos de sujeción forman una puntada bifurcada en el tejido (figura 2b). El cosido resultante explica la forma que toma el objeto resultando cestas firmes de paredes duras, de forma cónica y base plana (Figura $5 \mathbf{a})$.

\section{b) Materia prima.}

1. Identificación de las fibras xilemáticas.

En el Laboratorio de conservación de material arqueológico de origen orgánico de la CNCPC se obtuvieron seis muestras (Figura $2 \mathrm{~b}$ y Figura 5) de $2 \times 1 \mathrm{~mm}$ de las fibras xilemáticas que conforman la estructura (fundación y puntos de sujeción) de tres cestas y de un fragmento suelto de cestería pertenecientes a la Cueva de la Candelaria. En el 


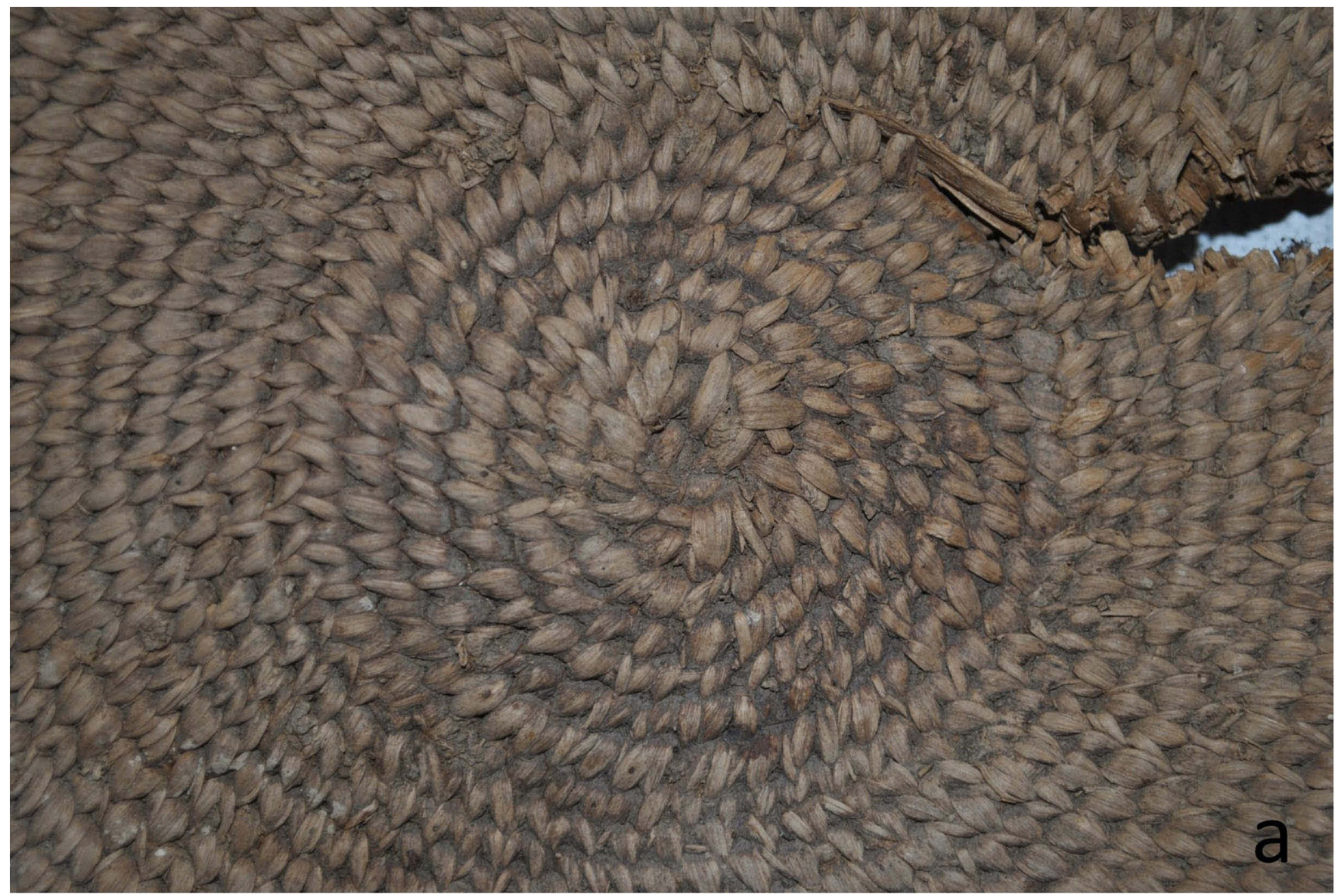

Figura 2a. Vista general de la cesta 10-423669 con tejido enrollado en espiral.
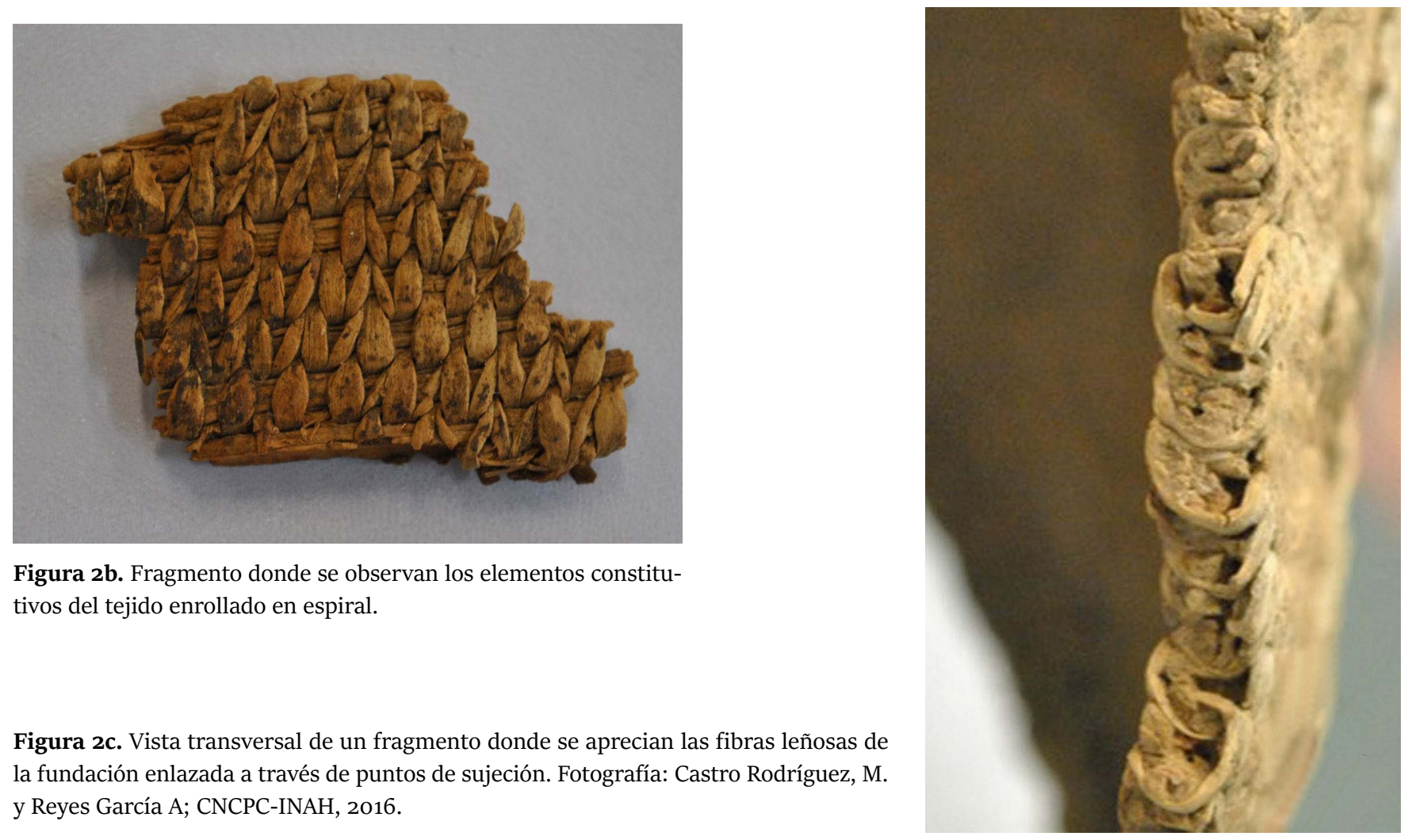

Figura 2c. Vista transversal de un fragmento donde se aprecian las fibras leñosas de la fundación enlazada a través de puntos de sujeción. Fotografía: Castro Rodríguez, M. y Reyes García A; CNCPC-INAH, 2016. 


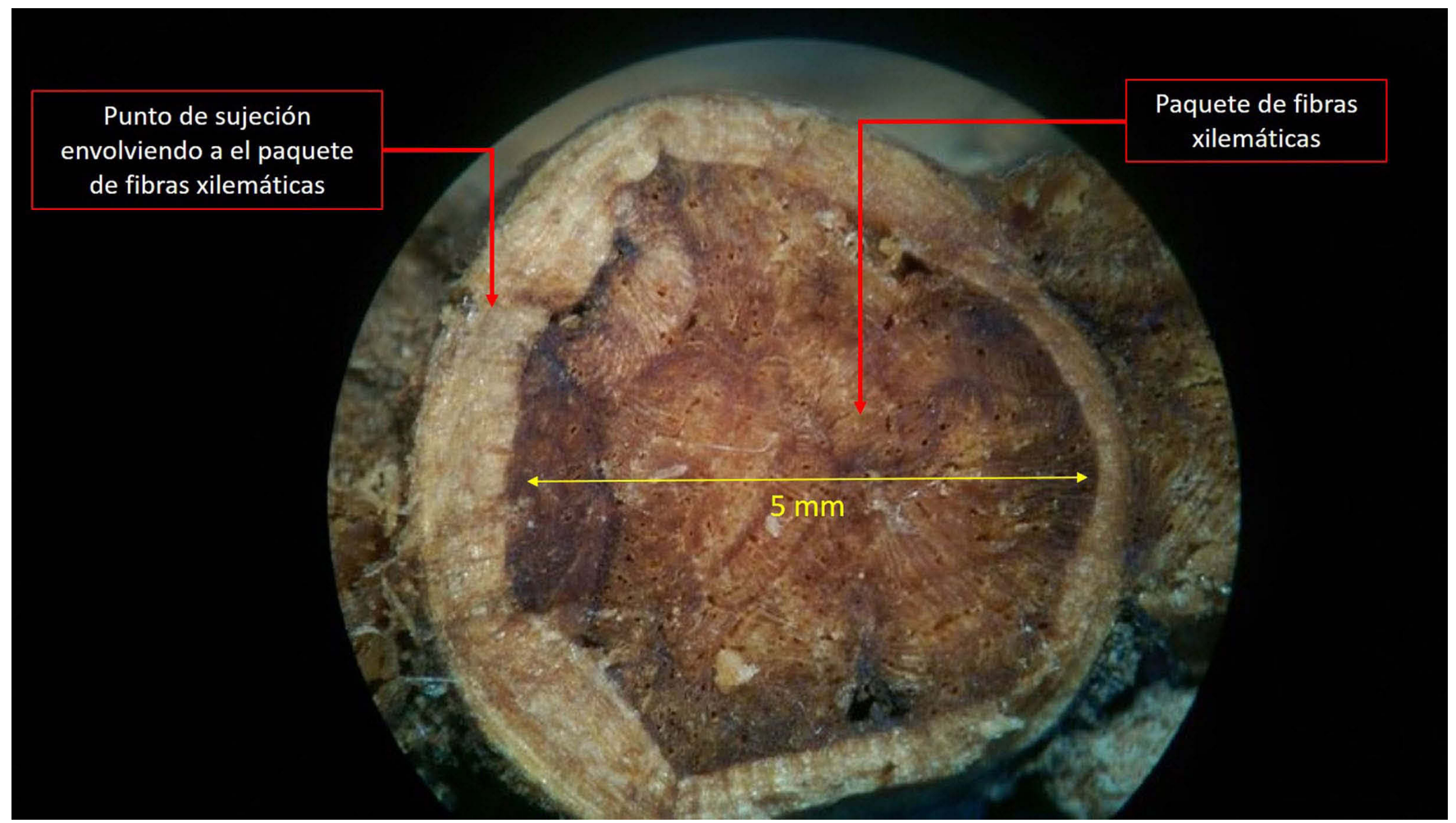

Figura 3. Sección transversal de la fundación que muestra haces xilemáticos sujetados con fibras en sentido longitudinal (40X). Fotografía: Dra. Alejandra Quintanar Isaías, Laboratorio de Anatomía Funcional y Biomecánica de Plantas Vasculares. Departamento de Biología -Universidad Autónoma Metropolitana Iztapalapa.

Laboratorio de anatomía funcional y biomecánica de plantas vasculares en la UAM-Iztapalapa se procesó el material muestreado. Las pequeñas muestras se hidrataron con agua e incluyeron en Tissue $T e c \AA$ para hacer secciones radiales, transversales y tangenciales del tejido usando un Criostato Leica a $-20^{\circ} \mathrm{C}$. Se hicieron laminillas permanentes del tejido vegetal. Se observaron los patrones celulares que componen el tejido y se describieron. La primera descripción del tejido arrojó que se trataba del xilema secundario (madera) de un género arbustivo perteneciente a la familia Euphorbiaceae.

\section{Trabajo de campo.}

Para hacer la identificación del género botánico de la madera se hizo un viaje de campo a la zona en el Valle de las Delicias. Se realizó un registro de los géneros representativos de la familia identificada en la zona. Se colectaron 15 ejemplares con raíces y tallos de estos géneros sobre las orillas de la carretera federal Bermejillo-Torreón, Coahuila, México.
En el laboratorio se siguió el mismo protocolo para preparar laminillas permanentes del tejido perteneciente a diversos géneros de la misma familia. Los patrones celulares que ajustaron a las fibras xilemáticas de las cestas fueron los del género Jatropha dioica, conocida en la región como Sangre de Drago, la que es una especie característica y presenta propiedades de elasticidad ad hoc para la elaboración de tiras finas, blandas y resistentes para tejer.

La identificación de las fibras ha dado luz acerca del avance tecnológico que representó en estos grupos humanos y su trascendencia a otros grupos que en la actualidad siguen usando estas plantas para la elaboración de cestas monumentales como es el caso de la Nación Comcaác ${ }^{4}$ en el estado de Sonora, donde usan el Torote (Jatropha cuneata), (comunicación personal Lic. Alejandrina Espinoza Reyna ${ }^{5}-$ Museo Comcaác). Ambas especies presentan patrones celulares correspondientes a los encontrados en las fibras xilemáticas de las canastas de las cuevas. 


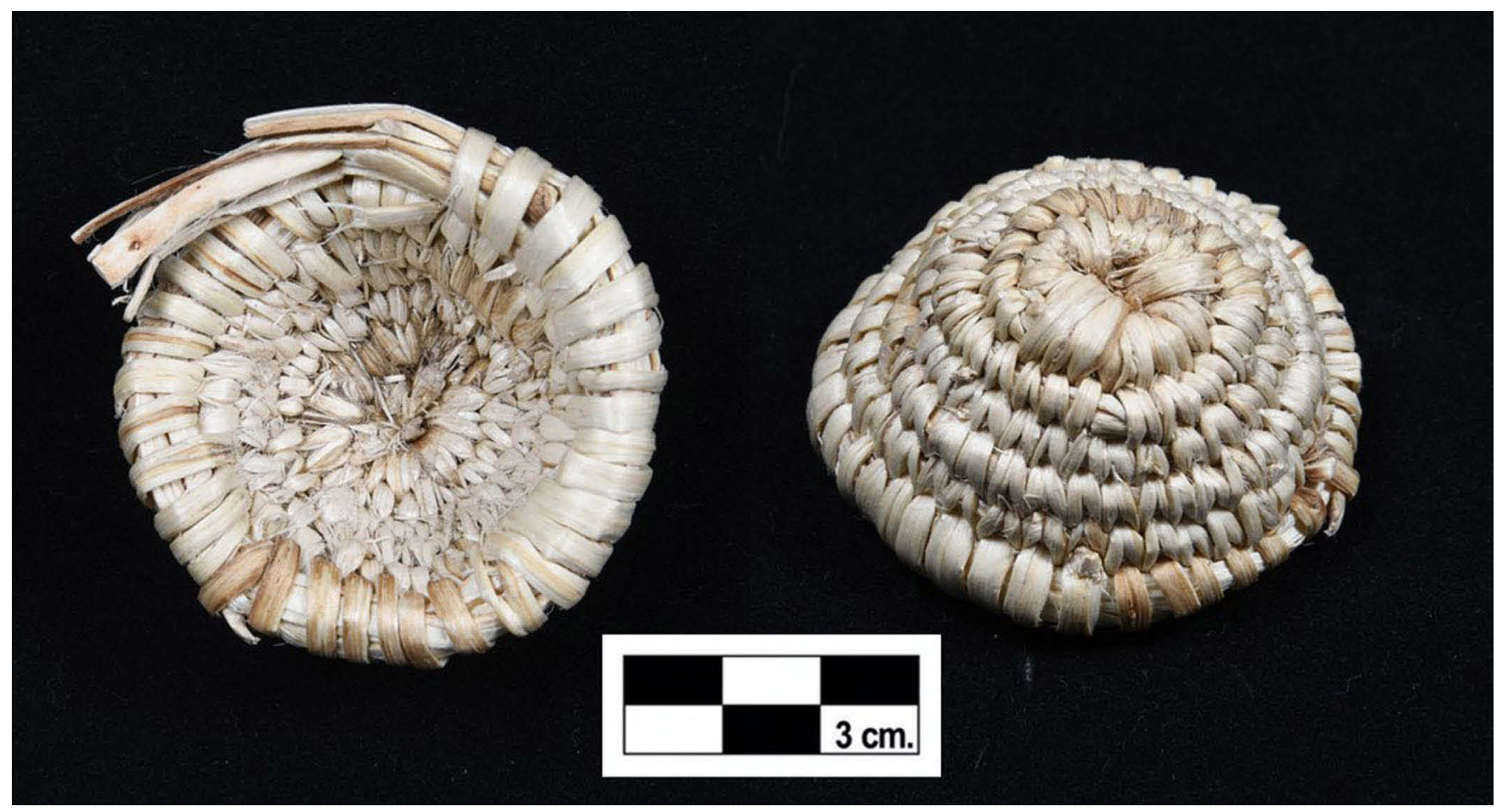

Figura 4. Cesta en proceso de elaboración siguiendo la técnica de enrollado en espiral con fibras de Jatropha dioica, vista general del interior y exterior. Fotografía: Julio Broniman, CNCPC-INAH 2020.

\section{c) Reproducción de la tecnología con fibras de sangre de drago.}

La reproducción de la técnica se realizó usando 12 plantas de sangre de drago de $20-30 \mathrm{~cm}$ de longitud.

\section{Eliminación de la corteza.}

Se plantearon tres procesos de manufactura para la eliminación de la corteza y la preparación de la fibra.

1) Eliminación de la corteza en verde. Cuatro tallos recién cortados se tallaron, sin embargo, la corteza quedó adherida. Fue difícil eliminarla y durante el tallado quedaron pedazos adheridos a la fibra xilemática.

2) Eliminación con pudrición de la corteza. Cuatro tallos se hidrataron en una tina con agua por una o dos semanas para que el tejido se pudriera sin lastimar las fibras.

En este método la corteza se eliminó fácilmente lavando con la mano, pero las fibras se deformaron. Con este proceso las fibras se secaron rápidamente. Las fibras quedaron quebradizas con rigidez y con dificultad para desprender "hebras o hilos" largos para tejer, dificultando su manipulación.
Adicionalmente las fibras que se obtuvieron eran cortas y algunas dañadas o podridas lo que las hizo perder flexibilidad.

3) Eliminación de la corteza con calor. Cuatro tallos se pusieron en anafres tradicionales con brasas recién prendidas. Los tallos se asaron para eliminar la corteza, tal como lo realizan los grupos Seris en Sonora (Alejandrina Espinoza, comunicación personal). Este método fue sencillo y rápido y se requirió poco calor para desprender. La corteza se eliminó fácilmente con la mano, las fibras quedaron lustrosas y flexibles; el cilindro de madera fue fácil de abrir o seccionar para jalar hilos o hebras largas. Estas últimas se emplearon para tejer o realizar las sujeciones de las fundaciones. Las fibras son fáciles de manipular y recuperan su flexibilidad cuando se hidratan.

2. Elaboración de la cesta con las fibras obtenidas con calor, siguiendo la técnica de enrollado en espiral.

La fundación se formó con las fibras obtenidas de la planta completa. Se conformó un haz de fibras de aproximadamente 5 hebras dando $5 \mathrm{~mm}$ de grosor; estos paquetes de fibras se enlazaron por medio de una sola hebra de fibras del xilema, a manera de hilo, siguiendo el método de enrollado en espiral. 


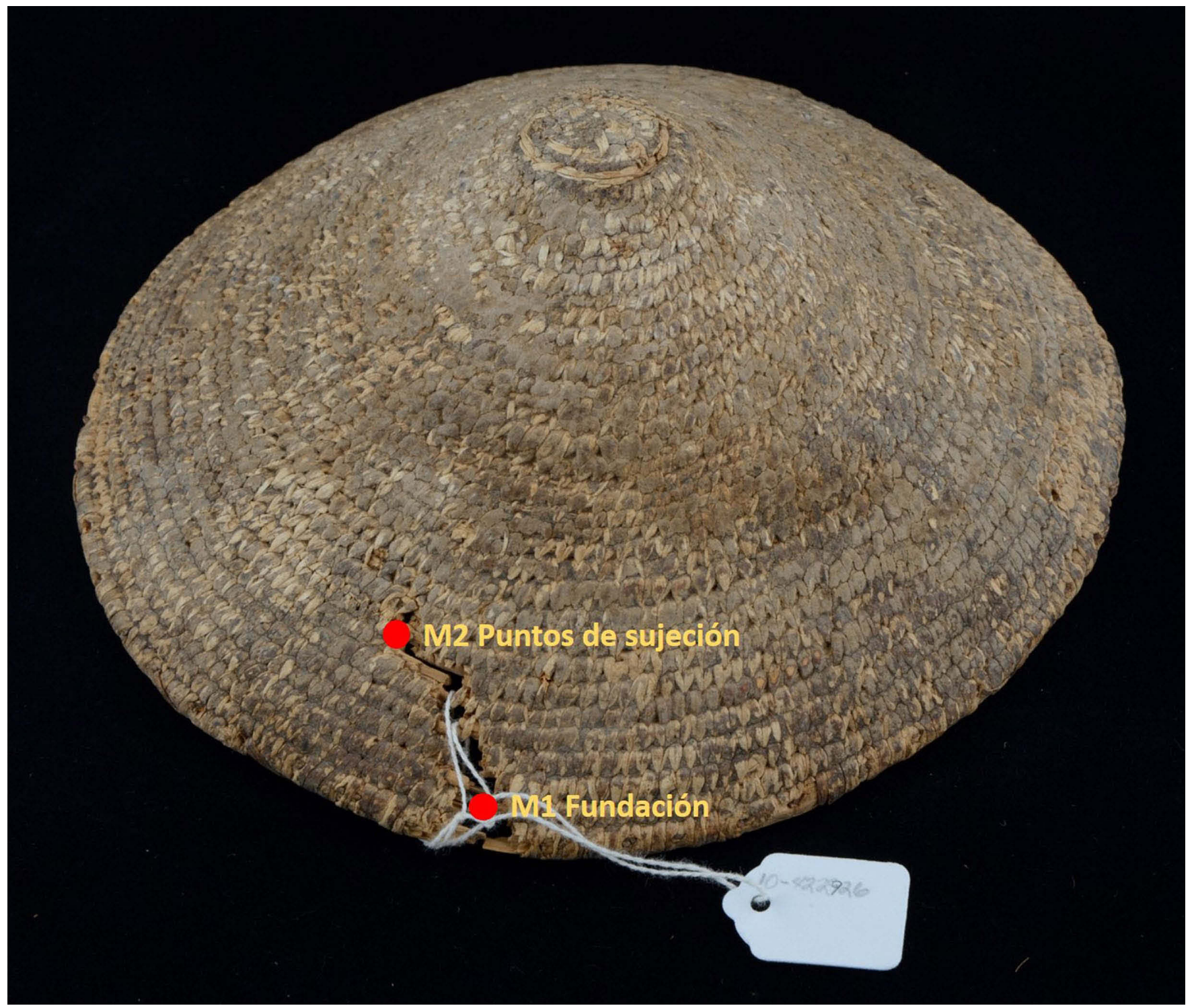

Figura 5. Cestas, antes de su intervención, en las que se ubica los sitios de extracción de las muestras. Fotografías: Julio Broniman, CNCPCINAH, 2015-2016.

La sujeción se logra enlazando la primera hilera de la fundación sobre la siguiente haciendo un orificio central en ambas fundaciones para que pase la puntada de forma envolvente, de manera consecutiva, hasta subir al siguiente nivel; dónde la puntada pasará por en medio del punto de sujeción anterior abriendo la hebra en forma de "V" como se observa en la figura $2 b$.

Para abrir o realizar el orificio que permite la sujeción se emplearon 2 tipos de espinas: una obtenida de la punta o espina de maguey y otra punta o espina de una planta de la región (Koeberlinia sp.).
La figura 4 muestra los resultados del proceso de elaboración de una cesta usando los materiales y la técnica ad hoc reproduciendo un objeto de aproximadamente $5 \mathrm{~cm}^{3}$, y que llevó alrededor de 7 horas de trabajo. Es posible que los grupos de nómadas recolectores del Norte de México podrían realizar estos objetos en mucho menor tiempo, con la maestría que con seguridad los caracterizaba.

La reproducción experimental de este objeto emplea la cocción como método de limpieza y preparación de la materia prima, esto permitió obtener fibras de excelente calidad. Este proceso es similar a lo que hoy en día realizan los artesanos de la Nación Comcaác en Sonora 

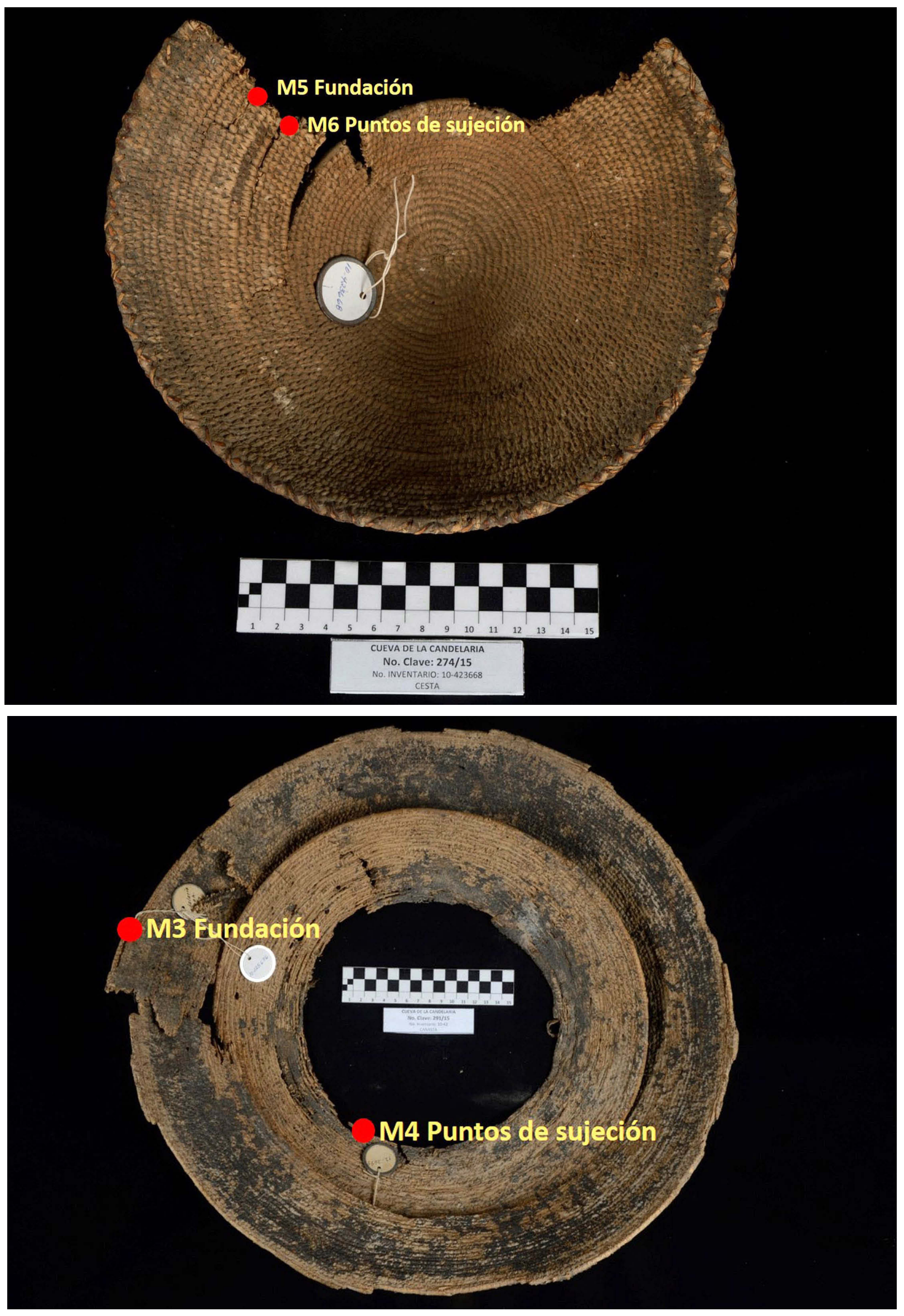


\section{Procesos de conservación y restauración de las cestas estudiadas}

Con respecto a los procesos de conservación y restauración, los bienes fueron intervenidos bajo los siguientes criterios de conservación:

- Respeto a la integridad del patrimonio cultural.

- Soluciones reversibles que permiten la retratabilidad de los materiales.

- Intervenciones reconocibles que se integran visual y estéticamente al bien cultural y que permiten su mejor comprensión a las generaciones actuales y futuras.

- Al ser patrimonio que forma parte de la colección de un museo se buscó resaltar la función didáctica y estética con la intención de proporcionar una visión clara del bien en la mente del interlocutor.

El MUREL cuenta con 62 registros de cestería en la base de datos; la mayoría de ellos son fragmentos y de las 15 cestas que se encuentran más completas, se han intervenido 7 cestas hasta el día de hoy.

Estos objetos experimentaron condiciones de enterramiento y almacenamiento desde su descubrimiento por lo que las fibras se encuentran sumamente resecas, lo que puede explicar la presencia de fisuras, grietas, roturas, deformaciones y pérdida de tejido o material.

Para iniciar el proceso de conservación las fibras se rehidrataron, lo que permitió la manipulación del objeto y ninguna pérdida del material. La hidratación flexibilizó las fibras de manera que se pudieron corregir las deformaciones $\mathrm{y}$ tratar las grietas y fisuras.

La consolidación se realizó paulatinamente usando derivados de polímeros naturales y semisintéticos: mezclas de polietilenglicol-almidón y methocel, en proporciones que se han desarrollado dentro del laboratorio de conservación arqueológica de material orgánico.

Para cerrar las grietas se emplearon injertos de bambú, así como refuerzos y retejidos de papel japonés, y finalmente, en los casos que fue necesario, se realizaron injertos removibles de resina epóxica con el fin de dar estabilidad a las piezas, y reintegrar la unidad estética al objeto.

\section{Conclusiones}

El grupo de cazadores recolectores que habitó en el valle de las Delicias, tenía un profundo conocimiento de su entorno.
Esto les permitió aprovechar los recursos presentes para la selección, recolección y transformación de los materiales.

De acuerdo con la base de datos de los bienes albergados en el MUREL se cuenta con aproximadamente 15 cestas cónicas, elaboradas con la técnica de espiral, de diversos tamaños, pero con las mismas características organolépticas.

El principal deterioro de los objetos se observa en los fondos, dónde la gran mayoría de éstos presenta pérdida de tejido, conservando sus paredes con grietas longitudinales y transversales.

La identificación anatómica microscópica de la planta sangre de drago fue fundamental para determinar la materia prima empleada en la elaboración de estos objetos, así como para experimentar en la reproducción de la manufactura y entender la tecnología cestera de este grupo de cazadores recolectores.

Las similitudes en las propiedades físicas y anatómicas de las fibras xilemáticas de Jatropha cuneata (Torote) y Jatropha dióica (Sangre de Drago), sugiere un aprovechamiento de las plantas del desierto basada en una tecnología ancestral preservada por los artesanos de la nación Comcaác.

El trabajo interdisciplinario permite el acercamiento al conocimiento de los materiales y técnicas que emplearon los antiguos mexicanos, para rescatar nuestra historia, promover y recuperar las tradiciones perdidas. En este ejemplo de interdisciplina, la Conservación no se centra únicamente en hacer perdurar los bienes culturales, la Botánica Estructural tampoco se reduce a la identificación de tejidos vegetales. La reproducción de objetos de la vida cotidiana de este grupo de cazadores recolectores se vuelve una vivencia completa que implica la reconstrucción del pensamiento y la práctica de estos grupos para entender cómo resolver los procesos de transformación de las materias primas para formular soluciones que expliquen el uso, en este caso, de plantas del desierto.

\section{Agradecimientos}

Queremos agradecer todo el apoyo brindado por parte de las restauradoras Adriana Reyes García y Miriam Elizabeth Castro Rodríguez para la elaboración de esquemas y tomas de muestras; así como a todo el equipo del Laboratorio de Anatomía Funcional y Biomecánica de Plantas Vasculares del Departamento de Biología -Universidad Autónoma Metropolitana Iztapalapa; a María Isabel Ambrosio Torres por su invaluable trabajo experimental con las fibras de la planta Sangre de Drago y especialmente a la Dra. Victoria Solanilla por sus atenciones, invitación y apoyo para la difusión del presente trabajo en la 8th Pre-columbian Textile Conference. 


\section{Fuentes consultadas}

Adovasio J. M.;

1977. Basketry Technology: A Guide to Identification and Analysis. Aldine Publishing Company. New York.

Aveleyra Arroyo de Anda, Luis; Maldonado-Koerdell, Manuel; Martínez del Río, Pablo;

1956. Cueva de la Candelaria, Vol. I, Memorias del Instituto Nacional de Antropología e Historia V, INAH-SEP, México.

1964. Sobre las fechas de Radiocarbono 14 para la Cueva de la Candelaria, Coahuila. Anales de Antrpología I, pp. $125-130$

González, Arratia Leticia;

1998. La cueva de la Candelaria, Arqueología Mexicana, Vol. 30, México.

1999. Museo Regional de la Laguna y la cueva de la Candelaria; CONACULTA-INAH, México.

2006. Las cuevas mortuorias de Coahuila; Arqueología Mexicana; Vol. XIV, Número 8o, pp. 61-65.

Johnson Weitlaner, I.;

1977. Los textiles de la Cueva de la Candelaria, Coahuila. Departamento de Monumentos Prehispánicos, Colección Científica, Arqueología, No. 51, INAH, México.
Mansilla Lory, Josefina; Pijoan Aguadé Carmen Ma.;

200o. Evidencia de treponematosis en la cueva de la Candelaria, Coahuila, con énfasis en un bulto mortuorio infantil; Chungara: Revista De Antropología Chilena, vol. 32, no. 2, pp. 207-210. www.jstor.org/stable/27802140.

200o. La Cueva de la Candelaria: Bultos Mortuorios y Materiales; Chungara: Revista De Antropología Chilena, vol. 32, no. 2, pp. 211-215

Pijoan Aguadé, Carmen Ma.; Mansilla Lory, Josefina;

200o. La cueva de la Candelaria: Bultos mortuorios y materiales; Chungara: Revista De Antropología Chilena, vol. 32, no. 2, pp. 211-215. www.jstor.org/ stable/27802141

Sánchez Valenzuela, Gloria Martha; Castro Rodríguez, Miriam Elizabeth; Reyes García, Adriana;

2017. Conservación de cestería en espiral, proveniente de la Cueva de la Candelaria, Torreón, Coahuila: criterios, tratamientos y líneas de investigación. Revista CR Conservación y Restauración, N11/12, abril-agosto 20017, pp. 115-129, Revistas INAH-CNCPC, CDMX. 